\title{
On Intermittent Control of Time Delay System with Actuator Saturation
}

\author{
Wu Jun \\ Electrical Engineering Department \\ Tianjin Metallurgical Vocation- technology Institute \\ Tianjin, China \\ E-mail: 397656413@qq.com
}

\begin{abstract}
With the development of science and industry, the rapid increase in time-delay systems has prompted more and more researchers to use intermittent control theory to analyze and synthesize the stability of the time-delay systems subject to actuator saturation and random perturbation. This note investigates the development status and research significance of time-delay systems, Intermittent control and actuator saturation, and that by method of Intermittent control analysis and discussion of the stability of time-delay systems with actuator saturation is of great theoretical significance and practical application value.
\end{abstract}

\section{Keywords-Intermittent control ;Time delay}

system;Actuator saturation

\section{A BRIEF INTRODUCTION TO TIME DELAY SYSTEM}

\section{AND ITS RESEARCH SIGNIFICANCE}

Time delay is also called detention or time detention. The dynamic system with time delay exists extensively in the nature and all the engineering fields, such as power system, network control system, financial system, biological system, bio-medicine, environment, chemical process, mechanical process, neurotic network, building structure, aerospace industry and all kinds of production lines. They are all typical delay systems. Time delay system appeals to many experts and scholars because its application is very general and extensive. Zhang Wenfeng and $\mathrm{Hu}$ Haiyan carried out in 2001 parameter identification studies on the non-linear dynamic control system with feedback time delay[1]; Iv'anescu. Niculescu et al discussed extensively applied neutral time delay system (steam-pipe and water-pipe system and heat-exchanger system)[2], focusing on the consistent asymptotic stability of linear neutral system, and obtained the stability conditions of time delay dependence; Singh and Singhose studied the real-time shaping control and time delay control of flexible structures[3], referring to the real-time shaping and time delay filtering; Richard analyzed all sorts of models caused by time delay, stability and structural changes and briefly introduced some controlling methods, including sliding mode control and time delay control; he also discussed the structural use of time delay input under specific sliding conditions to realize the distributive time delay control of numbers and to process the time delay values of relevant information; Dong Xiaojuan carried out in 2010 an in-depth study on the random resonance of asymmetric dual stability system containing noise and time delay[4], discussing the influence of random force on the time delay dynamic system by means of functional differential equations.

Time delay is caused by many factors, mainly including the parametric measuring delay in the system (the speed of measuring instruments), the speed of chemical reactions, the speed of data transmission by sensor, the correlation between long-pipe and belt transmission speed and the transmitted substance, etc. To sum up, time delay includes parametric measuring delay, launching delay, transmitting delay and processing delay. At present, it is not realistic to overcome or stifle all the time delays, therefore, it is utterly important to study the stability of time delay system and to make sure of the performance the system expects.

The changing tendency of continuous time delay system is not only associated with the state of the current moment, but it also depends on the state of a certain moment or certain moments in the past, so the continuous time delay system is a infinite dimension system and the characteristic equation of the system is a transcendental equation and contains an infinite number of values. As for the discrete time delay system, the dimension of the system changes with the time delay changing tendency, taking on geometrical rules, which increases the difficulty of the stability analysis of the system. Therefore, studies on both continuous and discrete time delay systems are fairly challenging. In addition, it is of great value for the application of actual systems.

\section{The Current State of Time Delay System}

From the perspective of systematic theories, any controlling system with time delay will be influenced by the past state of the system, so when studying time delay controlling systems, we should not only consider the 
system's current state. This is a direct reason of the fact that the solution of the time delay system's state equation is absolutely different from those of ordinary differential equations. Therefore, new ways of thinking, perceptions and methods should be made use of when we discuss the stability and performance of this kind of controlling systems. Liu Hetao further studied in 1986 algebraic criteria of time delay system's unconditional stability and transformed the unconditional stability into judging the characteristic values distribution of the matrix " $\mathrm{A}+\mathrm{B}$ " in the time delay system as well as some additional conditions[5]. Liang Jiaxiu and Chen Siyang analyzed and put forward in 2001 the criteria of the unconditional stability of the third-order time delay deferential equation[6]. Jacovitti and Scarano carefully analyzed the delay estimation of the time delay controlling system by means of time discretion method[7]. Hu Haiyan and Wang Zaihua analyzed in 1999 the studies on strategies, systematic features and hot issues of dynamics of the non-linear delay dynamic system[8].

Time delay system can be divided into two categories: continuous time delay system and discrete time delay system. Their state equations can be shown as follows:

$$
\begin{aligned}
& x(t)=A x(t)+B x(t-\tau), \tau \geq 0 x(t)=\theta(t), t \in\left[\begin{array}{ll}
-\tau, & 0
\end{array}\right] \\
& \&(t)=A x(t)+B x(t-d(t)), \quad d(t) \geq 0 \quad x(t)=\theta(t), \\
& \mathrm{t} \in[-\max [d(t)], 0](2) \\
& x(k+1)=A x(k)+B x(k-\tau), \tau \geq 0, x(k)=\theta(k), \quad k \in[-\tau, 0] \text { (3) } \\
& x(k+1)=A x(k)+B x(k-d(k)), d(k) \geq 0, x(k)=\theta(k), \\
& k \in[-\max [d(k)], 0](4) \\
& \mathcal{R}(t)=A x(t)+B_{1} x\left(t-\tau_{1}\right)+B_{2} x\left(t-\tau_{2}\right)+\Lambda+B_{n} x\left(t-\tau_{n}\right), \\
& \tau_{1}, \quad \tau_{2}, \quad \Lambda \quad \tau_{n} \geq 0 \\
& \left.x(t)=\theta(t), \mathrm{t} \in\left[\begin{array}{llll}
-\max & {\left[\tau_{1},\right.} & \tau_{2}, \Lambda, & \tau_{n}
\end{array}\right], 0\right](5)
\end{aligned}
$$

wherein "(1)" shows the continuous time constant delay system, "(2)" shows the continuous time time-varying delay system, correspondingly, “(3)" shows the discrete time constant delay system, “(4)" shows the discrete time time-varying delay system. "(5)" shows a model of multiple delay continuous time system. At present, studies on time delay systems mainly includes system's stability analysis, controller design, $H_{\infty}$ performance analysis, $H_{\infty}$ filter design, observer design and so on.

\section{SATURATION PHENOMENON}

The phenomenon of actuator saturation (as is shown in Fig .1, there are two kinds of saturation, the dotted lines describe non-standard saturation while the solid lines describe standard saturation) exists extensively in the nature and all kinds of systems, such as aerospace industry, mechanical manufacturing process, bio-system, network control system, power system and chemical production. On one hand, saturation stems from the limitation of the actuator's own physical features; on the other hand, it is introduced on purpose to prevent excessive charge from damaging the system's actuating institution and stability. Therefore, studies on actuator saturation are of great theoretical and practical significance. The actuator saturation may not only decrease the system's performance, but also destroy the system's stability. Therefore, it is quite necessary carry out studies on control systems with actuator saturation. Domestic and foreign experts often deal with saturation by means of two strategies: the first one is to design the controller according to linear system without considering saturation and then introduce compensation links to set off the negative influences that saturation brings to the system. This is so-called Anti-windup method, also called indirect method; the second one is to consider saturation and non-linear way and carry out studies by means of non-linear theories when designing the controller. This is direct method.

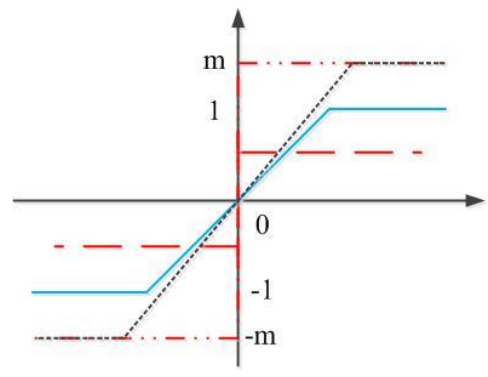

Figure 1. Schematic Diagram of Actuator Saturation

Since the problem of actuator saturation exists extensively in our daily life and practical engineering and it is urgent to deal with and make use of saturation and non-linear method, it draws much attention of many domestic and foreign scholars. Chen Jiaorong and Liu Feiji carried out studies on the predictive control of hopping system containing actuator saturation based on the Robust model[9]; Chen Wuhua, Li Danxia and Cao Dunqian discussed the stability of linear impulse system containing input saturation[10]; Wang Juan, Liu Zhiyuan and Pei Run carefully analyzed in 2008 the attraction domain of saturated linear system and put forward an improved estimation algorithm; Raouf and Boukas discussed the stabilization of discrete time general Markovian hopping system containing input saturation and switching.

\section{A BRIEF INTRODUCTION TO INTERMITTENT}

\section{CONTROL}

The intermittent control can be simply described as a kind of special segmented control, that is to exert control in a certain period of time in every control cycle and not to 
exert control in the rest of time in the control cycle, as is shown in Fig .2 (in every cycle $\mathrm{T}$, control signals are exerted in the 0 -h period and no control signal is exerted in the $\mathrm{h}$ - $\mathrm{T}$ period). The intermittent control can also be regarded as a transition between impulse control and continuous feedback control. In order to analyze a system with intermittent control, the state of system can be divided into two parts: free developing state and synchronous state. The free developing state corresponds to the period without external control, which is called autonomous cycle; the synchronous state corresponds to the period with external control, which is called control width. The intermittent control has many advantages in practice, for instance, it may stifle the system's spiral waves and it may help to solve the problems of chaos and hyper-chaos that seriously affect the system's stability. And the index stability problems of time delay chaotic systems can be solved by means of synchronous intermittent coupling of main and accessory tracks. In addition, the intermittent control is extensively used to reduce network jams and to guarantee the performance of network control systems. The intermittent control is a direct and efficient engineering method in terms of the common controlling process.

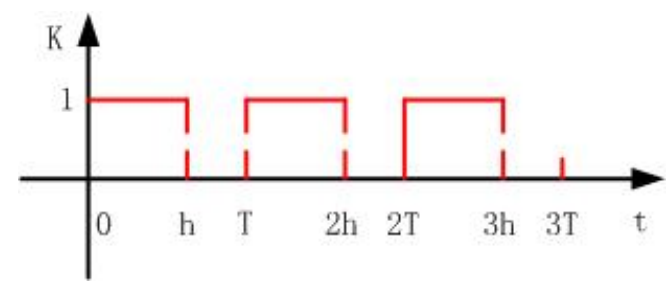

Figure 2. Schematic Diagram of Isochronic Intermittent Control

As a special form of switching control, the intermittent control can be divided into two categories: dependence switch (event switch) and time switch. The former one means that only when the state enters a preset particular area could the control be actuated (the particular area can be seen as a predefined event); the latter form of switch refers to the actuation of control signals in a certain limited interval and the system's state evolves freely beyond the intervals (autonomous period). Therefore, the control system is open within the autonomous cycle. As a special example of intermittent control, the impulse control draws more and more attention. An obvious feature of impulse control is that the state of the control system "hops" in a particular discrete moment, that is to say, the impulse control has zero duration. Since the state of the direct control system changes instantaneously, the impulse control is a more effective method when the system's state is observable; but the impulse control is not applicable when the state of the control system is not observable.

Compared with the traditional continuous control method, the intermittent control is easier and more effective because the system's output is intermittent, that is to say, the output parameters are not continuously measured. In order to realize different application aims and the system's different performance goals, intermittent control has been extensively applied to many engineering fields, for instance, all kinds of manufacturing, traffic and transportation, air-quality control as well as communication. It is also used to solve problems of constant system, singular system and non-linear system and to describe the asymptotic stability, overall stability, Robust stability, exponential stability, mean square stability, stochastic stability, cost-guaranty control, systematic reliability and stochastic control of chaotic systems in power engineering, physics and biological medicine and the $H_{\infty}$ performance, $H_{2}$ performance, $L_{2}$ performance and $L_{\infty}$ performance of different control systems and also design system's controller, full-order state observer, reduced order state observer, Kalman filter and $H_{\infty}$ filter.

In conclusion, time delay and actuator saturation are phenomena frequently met with in practical engineering and the discussion of systems simultaneously containing the two non-ideal elements meets not only practical needs but also theoretical values. The application of intermittent control to actuator saturation and the stability of time delay system may reduce the system's controlling cost and improve the actuator's controlling efficiency. Therefore, it is of great theoretical significance and practical value to analyze and discuss the actuator saturation and the stability of time delay system by means of intermittent control.

\section{REFERENCES}

[1] Wenfeng Zhang, Haiyan Hu. Parameter Identification of Non-linear Dynamic System with Feedback Time Delay, Journal of Vibration Engineering, 2001, 14(2): 314-318

[2] Ivănescu D, Niculescu S I, Dugard L, et al. On delay-dependent stability for linear neutral systems, Automatica, 2003, 39(2): 255-261

[3] Singh T. and Singhose W. Tutorial on input shaping / time delay control of maneuvering flexible structures, American Control Conference, 2002. Proceedings of the 2002, 3: 1717-1731

[4] Xiaojuan Dong. Random Resonance of Asymmetric Dual Stability System Based on Periodical Mixed Signals and Time Delay, Journal of Applied Mathematics of Controlling University, 2010, 25(2): 127-133

[5] Hetao Liu, a class of delay differential systems of algebraic unconditional decision, control theory and applications, 1986,3 (1): 106-110

[6] Jianxiu L, Chen Y, found the three order delay differential equations with unconditional stability, Shanxi Normal University Newspaper, 2001, 29 (1): 12-1 
[7] Jacovitti G. and Scarano G. Discrete time techniques for time delay estimation, IEEE Transactions on Signal Processing, 1993, 41(2): 525-533

[8] Haiyan $\mathrm{Hu}$, Wang Zaihua, research progress, nonlinear delay dynamic system advances in mechanics, 2002, 29(4):501-512
[9] Jiaorong C., Fei L, has a robust model of actuator saturation jump system predictive control, system engineering and electronic technology, 2008, 30 (4): 696-699

[10] Wuhua C, Danxia L, Dunqian C, the robust exponential stability of linear impulsive systems with inputsaturation of the,Journal of Guangxi University For Nationalities, 2011, 17 (1): 68-71 